\title{
Multicompartment Microparticles of SBT Triblock Terpolymers through 3D Confinement Assembly
}

\author{
Xiaolian Qiang ${ }^{1}$, Steffen Franzka ${ }^{2}$, Xuezhi Dai ${ }^{1}$, André H. Gröschel*,1 \\ ${ }^{1}$ Physical Chemistry and Center for Soft Nanoscience (SoN), University of Münster, 48149 Münster, \\ Germany. \\ ${ }^{2}$ Interdisciplinary Center for Analytics on the Nanoscale (ICAN), University of Duisburg-Essen, \\ 47057 Duisburg, Germany. \\ E-mail: andre.groeschel@uni-muenster.de
}




\section{SUPPORTING FIGURES}

a)

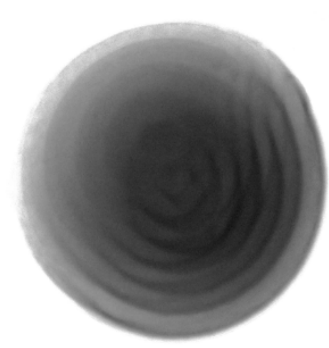

$100 \mathrm{~nm}$

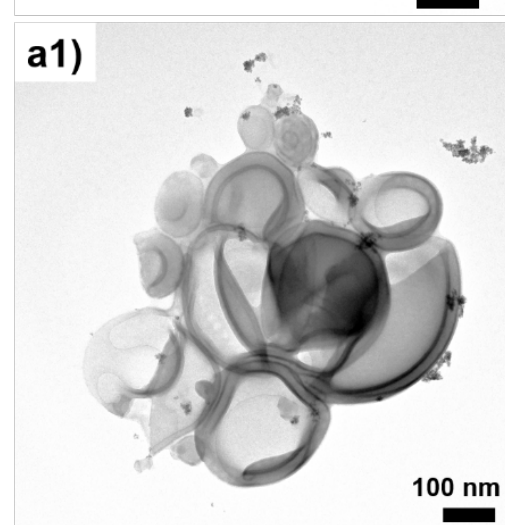

b)

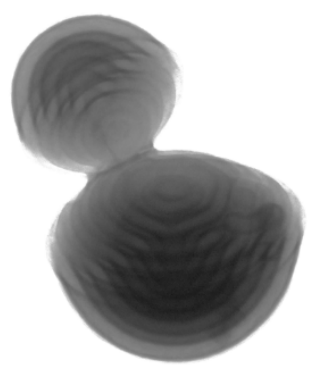

$100 \mathrm{~nm}$

b1)

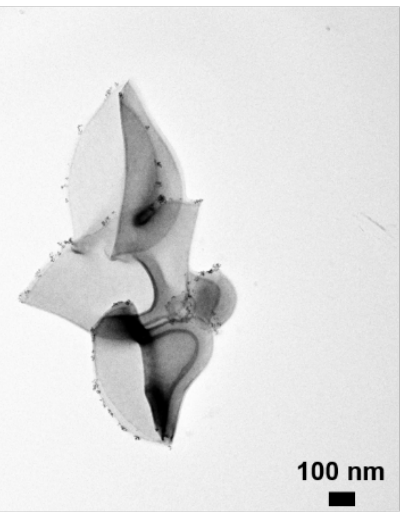

c)

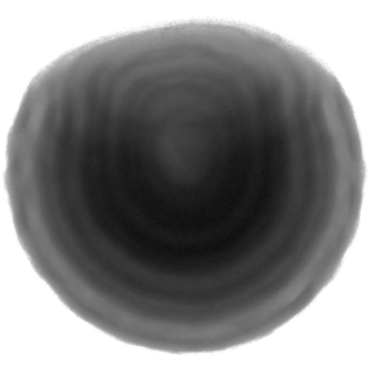

$100 \mathrm{~nm}$

c1)

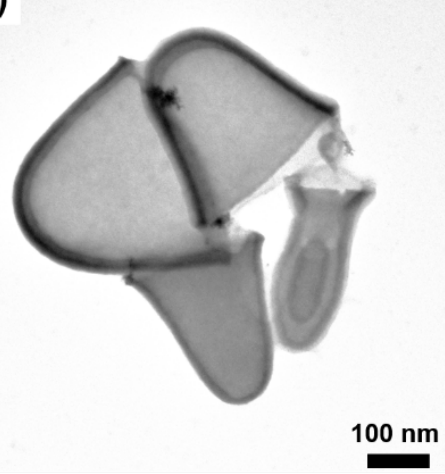

Figure S1. Lamella-lamella (LL) morphology in tulip-bulb microparticles. a, b, c) TEM images of $\mathrm{S}_{36} \mathrm{~B}_{20} \mathrm{~T}_{44}{ }^{148 \mathrm{~K}}, \mathrm{~S}_{38} \mathrm{~B}_{21} \mathrm{~T}_{41}{ }^{140 \mathrm{~K}}$ and $\mathrm{S}_{33} \mathrm{~B}_{43} \mathrm{~T}_{24}{ }^{94 \mathrm{~K}}$. a1, b1, c1) Disassembled structures after crosslinking $\mathrm{S}_{36} \mathrm{~B}_{20} \mathrm{~T}_{44}{ }^{148 \mathrm{~K}}, \mathrm{~S}_{38} \mathrm{~B}_{21} \mathrm{~T}_{41}{ }^{140 \mathrm{~K}}$ and $\mathrm{S}_{33} \mathrm{~B}_{43} \mathrm{~T}_{24}{ }^{94 \mathrm{~K}}$ with $\mathrm{OsO}_{4}$ and redispersion in THF. PB appears dark from $\mathrm{OsO}_{4}$ staining and $\mathrm{PS}$ grey. 
a)

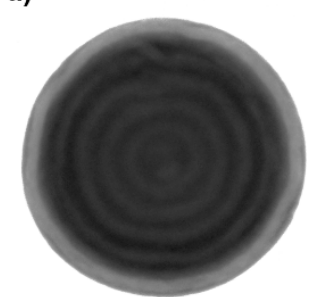

a1)

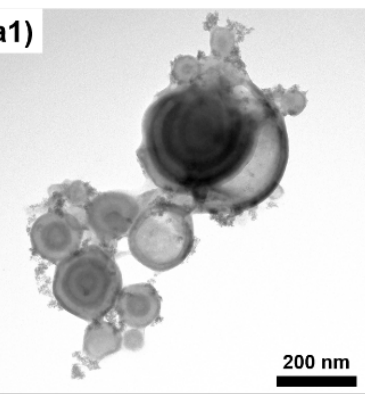

e)

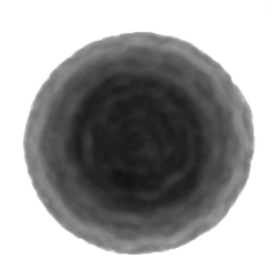

$100 \mathrm{~nm}$

e1)

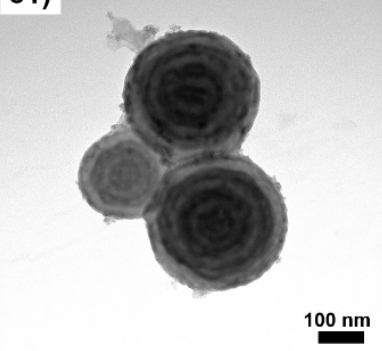

b)

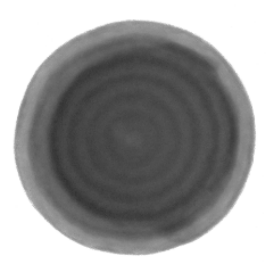

$100 \mathrm{~nm}$

b1)

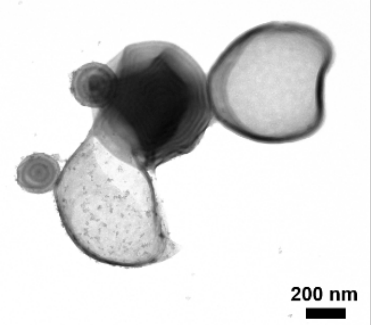

f)

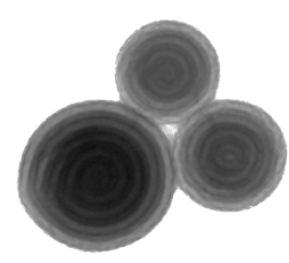

$200 \mathrm{~nm}$

f1)

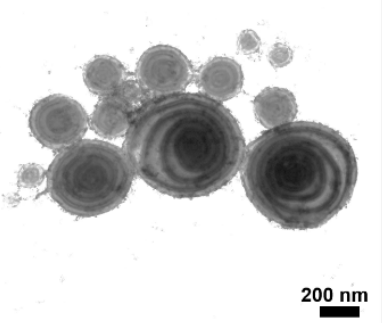

c)

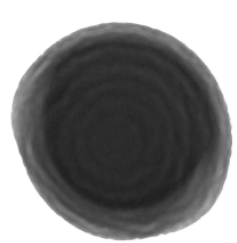

$100 \mathrm{~nm}$

c1)

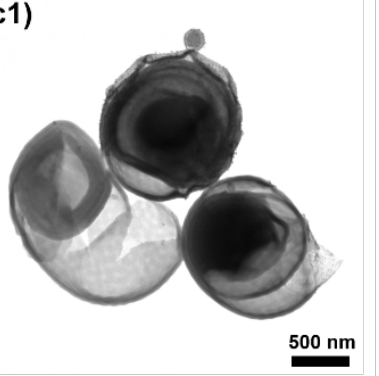

g)

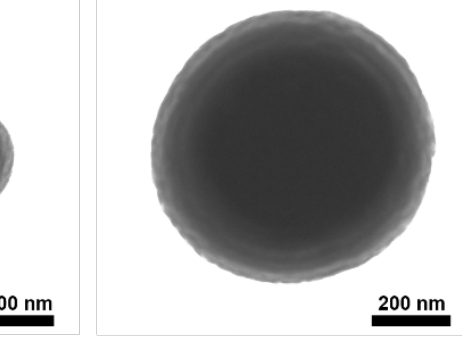

g1)

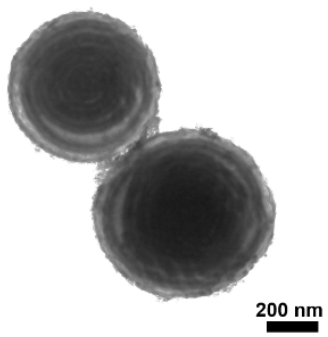

d)

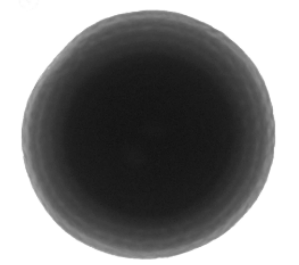

$200 \mathrm{~nm}$

d1)

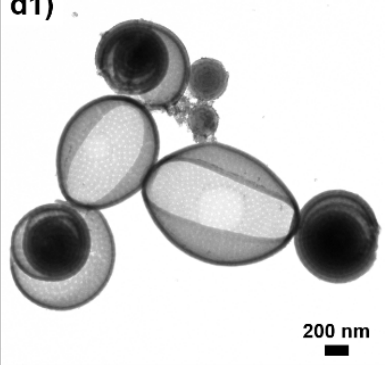

$00 \mathrm{~nm}$
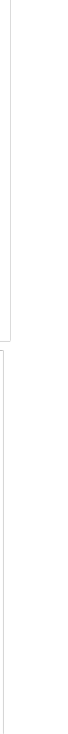

Figure S2. Concentric sphere-in-lamella (CSL) morphology in onion-like microparticles. a, b, c, d, e, f, g) TEM images of the $\mathrm{S}_{50} \mathrm{~B}_{45} \mathrm{~T}_{5}{ }^{64 \mathrm{~K}}, \mathrm{~S}_{56} \mathrm{~B}_{37} \mathrm{~T}_{7}{ }^{57 \mathrm{~K}}, \mathrm{~S}_{39} \mathrm{~B}_{51} \mathrm{~T}_{10}{ }^{80 \mathrm{~K}}, \mathrm{~S}_{53} \mathrm{~B}_{34} \mathrm{~T}_{13}{ }^{61 \mathrm{~K}}, \mathrm{~S}_{46} \mathrm{~B}_{41} \mathrm{~T}_{13}{ }^{69 \mathrm{~K}}$, $\mathrm{S}_{45} \mathrm{~B}_{40} \mathrm{~T}_{15}{ }^{71 \mathrm{~K}}$ and $\mathrm{S}_{36} \mathrm{~B}_{47} \mathrm{~T}_{17}{ }^{87 \mathrm{~K}}$. a1, b1, c1, d1, e1, f1, g1) Disassembled structures after crosslinking $\mathrm{S}_{50} \mathrm{~B}_{45} \mathrm{~T}_{5}{ }^{64 \mathrm{~K}}, \mathrm{~S}_{56} \mathrm{~B}_{37} \mathrm{~T}_{7}{ }^{57 \mathrm{~K}}, \mathrm{~S}_{39} \mathrm{~B}_{51} \mathrm{~T}_{10}{ }^{80 \mathrm{~K}}, \mathrm{~S}_{53} \mathrm{~B}_{34} \mathrm{~T}_{13}{ }^{61 \mathrm{~K}}, \mathrm{~S}_{46} \mathrm{~B}_{41} \mathrm{~T}_{13}{ }^{69 \mathrm{~K}}, \mathrm{~S}_{45} \mathrm{~B}_{40} \mathrm{~T}_{15}{ }^{71 \mathrm{~K}}$ and $\mathrm{S}_{36} \mathrm{~B}_{47} \mathrm{~T}_{17}{ }^{87 \mathrm{~K}}$ with $\mathrm{OsO}_{4}$ and redispersion in THF. PB appears dark from $\mathrm{OsO}_{4}$ staining and PS grey. 